doi: 10.3176/arch.2014.2S.13

\title{
12. DIE WIRTSCHAFTLICHEN GRUNDLAGEN DER SIEDLUNGEN DER ASVA-GRUPPE
}

In der Forschung herrscht bislang Einigkeit darüber, dass sich die agrarische Nahrungsmittelproduktion vornehmlich aus dem Bereich der Viehzucht und weniger dem Ackerbau zusammensetzte. Das vorhandene, vom Naturraum verfügbare Agrarpotential muss eine ausreichende Subsistenz gesichert und darüber hinaus die Grundlage für die in diesen Siedlungen zu beobachtenden Spezialisierungstendenzen im verarbeitenden Sektor geschaffen haben, etwa im Metallguss, in der Verarbeitung der Agrarprodukte und der Geräteherstellung (Knochen- und Geweihschnitzerei). Es scheint jedoch bezeichnend zu sein, dass im Ostbaltikum vor der Periode der Spätbronzezeit zwischen Küsten- und Binnenland regionale Differenzen in den ökonomischen Strategien und Ausrichtungen festzustellen sind. Solche permanent bewohnten Plätze wie Asva, dorfähnliche, kompakte Anlagen mit mehreren Gebäuden, basierend auf agrarischen Aktivitäten und solchen, die nicht unmittelbar mit der Nahrungsmittelbeschaffung zusammenhängen (Bronzeguss), werden im Ostbaltikum erst mit der Spätbronzezeit auf der archäologischen Bildfläche sichtbar. Synchron zur Entstehung der , befestigten' Siedlungen, der Wehrcharakter ist zumindest für einige estnischen Plätze fraglich (siehe Kap. 5), hat sich auch in der Entwicklungsgeschichte der Landwirtschaft eine neue Phase eingestellt. Veränderungen und Neuerungen bezüglich der Agrartechniken, der Erntemethoden und der gesamten Arbeitsorganisation scheinen nun stärker denn je auf Vorrätelagerung und Überschüsse ausgerichtet gewesen. Diese nach und nach zunehmend unabhängigere Lebensweise der Bronzezeitmenschen von den sie umgebenden natürlichen Risikofaktoren ermöglichte eine langfristig sesshafteortsgebundene Siedlungsweise und wird sich auch verändernd auf die Gesellschaftsverhältnisse ausgewirkt gehabt haben. Neueren Theorien zufolge markieren die Siedlungen vom Asva-Typ eine Entwicklung hin zu Territorialität und Besitzrecht in Verbindung mit Land und Ressourcen, insbesondere den für Viehzucht und Feldbau nutzbaren Bodenflächen (siehe unten). Man geht davon aus, dass sich auf Saaremaa und in der nordestnischen Küstenzone verschiedene nebeneinander existierende Siedlungs- und Wirtschaftsareale gebildet haben, mit sog. Zentralplätzen als Leit- und Verteilerstellen, denen die Kontrolle über Landbesitz, Nahrungsmittelund Güterproduktion oblag. Unter der Vorannahme dieses in der jüngeren Forschung allgemein akzeptierten Modells, sieht man für das Ostbaltikum der Bronzezeit eine vergleichsweise stark gegliederte Gesellschaft gegeben, mit der Verortung 
von Eliten in den Zentralplätzen - wie jenen Siedlungen der Asva-Gruppe. Im archäologischen Befundbild indes lassen sich sozial privilegierte oder elitäre Schichten oder Gruppen nicht ohne weiteres erkennen und vieles deutet lediglich auf gemeinschaftlich organisierte Hauswirtschaften hin, in denen Nahrungsmittelzubereitung, Sekundärverarbeitung agrarischer Produkte und selbst Bronzeguss separat praktiziert wurden. Diese Art von Siedlungs- und Wirtschaftsorganisation setzt zwar eine mehr oder weniger zentrale gesellschaftliche Führung voraus, doch bedarf es einer kritischen Bewertung gewisser sozioökonomischer Strukturmodelle (siehe dazu Kap. 13). Auch die wirtschaftliche Rolle des Robbenfangs in Asva und Ridala sollte näher beleuchtet werden.

Die Inselregion Saaremaa erweist sich hierbei als ein besonderer Naturraum, mit anderen Bedingungen und Voraussetzungen für Agrikultur als in Küsten- und Festlandszonen des heutigen Estland und Lettland. Zugleich haben Erforschung und Rekonstruktion der frühen Agrargeschichte im Inselraum Saaremaa nicht die gleiche Entwicklung erfahren wie auf dem estnischen Festland. Dort fehlt es noch an Datenerhebungen aus Bereichen der Archäobotanik (Pollenanalysen) und der Siedlungsarchäologie. Die Siedlungsfunde (z. B. das Gerätespektrum) und der Schlacht- und Speiseabfall der Wohnplätze der Asva-Gruppe liefern reichlich Hinweise bezüglich der Aktivitäten im Agrarsektor und im wirtschaftlich mindestens gleichbedeutsamen Bereich des Robben- und Fischfangs.

\subsection{Ackerbau}

Die markanten Veränderungen und Entwicklungen auf dem landwirtschaftlichen Sektor im bronzezeitlichen Saaremaa sind vornehmlich anhand naturwissenschaftlicher Quellen der Paläoethnobotanik dokumentiert. Derzeit am besten lassen sich mehr oder weniger gravierende, menschlich ausgelöste Eingriffe in die Vegetation der bronzezeitlichen Insellandschaft anhand einiger Pollendiagramme ablesen (Kaali, Pelisoo, Surusoo und Vedruka). Anzeiger für offene Wiesen, Ruderalflora und Waldrodung zeichnen sich dort deutlich ab. Auch die Entstehung der nährstoffreichen Alvar-Böden wird indirekt mit der während der Bronzezeit zunehmenden Viehweidung in Verbindung gebracht (Abb. 120) (Poska \& Saarse 1996, 157 ff., Tab. 1). Zwar mangelt es noch an ausreichend archäologischen Daten für die Jahrhunderte unmittelbar vor dem Einsetzen der Siedlungen der Asva-Gruppe, doch sprechen zumindest die anthropogenen Kulturanzeiger (und apophyten Gewächse) für eine stetige Entwicklung auf dem Agrarsektor. Die Pollendiagramme der Region reagieren ab dem ausgehenden Spätneolithikum mit zunehmenden Anzeigern für Brandrodung und Kultivierung (Poska \& Saarse 2002, 560, 566 f., Abb. 4, 10). Dabei zeichnet sich tendenziell eine menschliche Aktionsverlagerung in Inlandszonen der Insel ab, dies vermutlich im Zusammenhang mit fertileren Böden.

Für Saaremaa werden in einigen Pollendiagrammen (z. B. Pitkasoo) bereits gewisse Ackerbauaktivitäten im Übergangszeitraum vom Atlantikum zum Sub- 


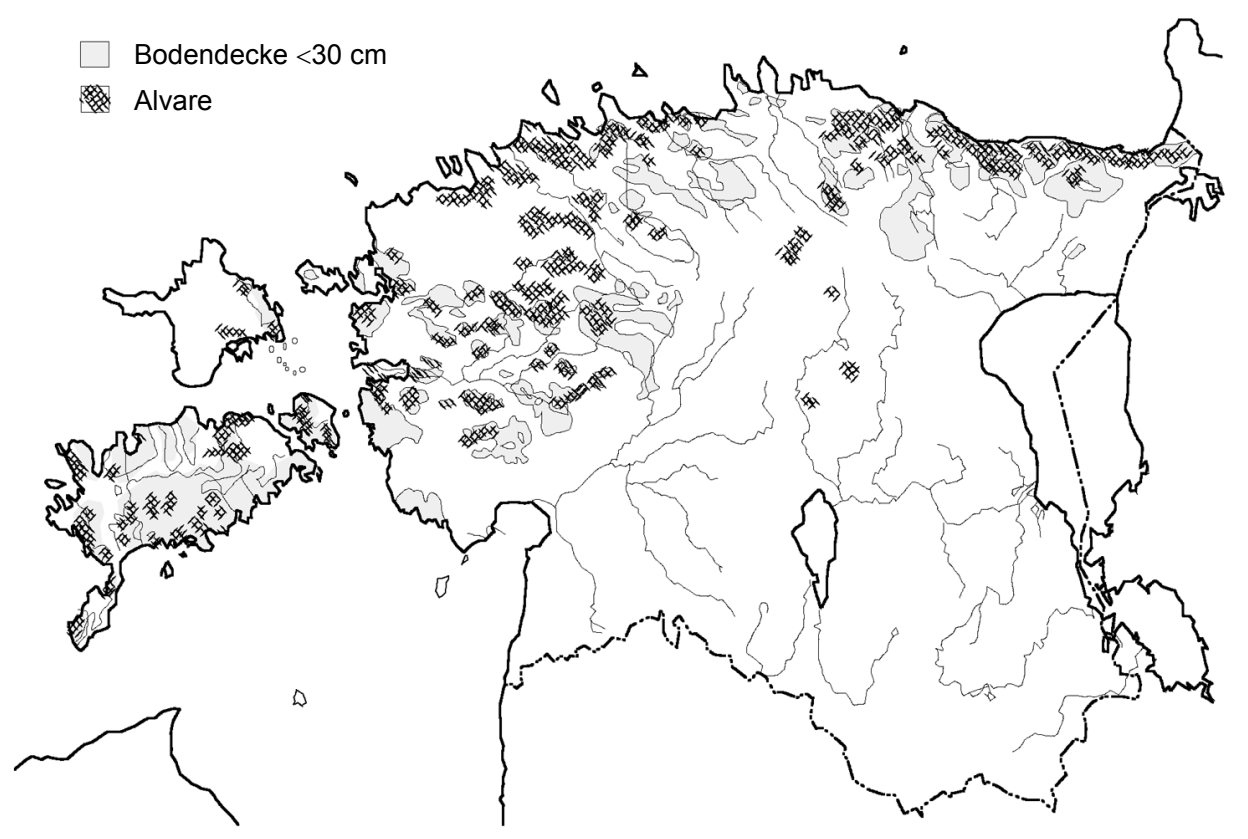

Abb. 120. Landschaften West- und Nordestlands mit dünner Bodendecke und für Ackerbau geeignete Alvare (estn. loo; nach Raukas 1995, Abb. 61).

Boreal bzw. im Frühneolithikum erkennbar (Saarse \& Königsson 1992; Lang 1995a, $130 \mathrm{ff}$.). Nach den Pollenspektren sind es gewisse Wiesen- und Weidelandanzeiger, die ein stetiges Lichten der Waldflächen signalisieren. Vereinzelten Nachweisen von Kulturpflanzenresten in der Frühphase des Neolithikums zufolge sollen dann erste zaghafte Versuche des Bodenbaus unternommen worden sein. ${ }^{202}$ Eine weitere Zunahme der Getreideanzeiger ist für das dritte vorchristliche Jahrtausend zu beobachten, und den Pollendiagrammen zufolge sollen sich in Estland Ackerbau und Viehzucht ungefähr zeitgleich mit der Schnurkeramik- und Bootaxtkultur etabliert haben (Saarse \& Königsson 1992; Poska \& Saarse 1996, 154 ff.; Lang 2003, 136 f.). Des Weiteren gibt es Anzeichen dafür, dass sich im Ostbaltikum im Zuge des Spätneolithikums die Subsistenz und Ressourcennutzung regional differenziert in den Küsten- und Inlandszonen entwickelte. ${ }^{203}$

\footnotetext{
202 Jüngst sind Zweifel an der Zulässigkeit pollenanalytischer Nachweise von Cerealia-Typen laut geworden, wegen eventueller Fehldeutungen von Wildgraspollen oder Probenkontaminationen (siehe Behre 2007, 208). Früher, primitiver Getreideanbau in ostbaltischen Siedlungsregionen (Lettland, Litauen) soll bereits im Mittelneolithikum praktiziert worden sein (Lang 1999, 364 f.; Bērziņš 2008, 371 f., 403 f.). In Finnland datieren die frühesten Belege merklich später, erst in das ausgehende Neolithikum.

203 Dazu Lang 1999, 366 f.
} 
Die archäologischen Forschungen in Asva haben bereits frühzeitig direkte und indirekte Hinweise für den örtlichen Getreideanbau zutage gebracht. Indreko (1939b, 26 ff.) machte auf steinerne Mahlwerkzeuge (Läufer, Reibsteine etc.; siehe Kap. 10.1) und auf Abdrücke von Getreidekörnern an der Asva-Keramik aufmerksam (evtl. Weizenart). Mit Ackerbau in Verbindung gebracht wurden auch knöcherne Geräte mit Lochung und einseitiger Zahnung, die konventionell als sog. Flachshecheln gedeutet werden. Allerdings konnte sich nicht bewahrheiten, dass Flachs oder Lein $\mathrm{zu}$ dieser Zeit in dieser Gegend überhaupt angebaut wurde. ${ }^{204}$ Weitere Getreideabdrücke auf Keramik wurden als solche von Gerste, Hafer und (vermutlich) Weizen identifiziert (Jaanits et al. 1982, 156, Abb. 107; Lang 2007b, 111 f.). Dass diese und andere Getreidearten tatsächlich genutzt wurden, zeigen die Makroreste aus lettischen Bronzezeitsiedlungen (z. B. Kivutkalns; Graudonis 1989, 106): verkohlte Körner von Weizen, Gerste, Hafer, Hirse sowie von Erbsengewächsen. Angetroffen im Fundmaterial von Asva, Ridala und Iru wurden auch mehrere mit dem Feldbau in Verbindung gebrachte Geräte aus Elchgeweih. Form, Größe und Zurichtung diese interessanten Artefakte lassen zwischen einfachen Hacken, Psalien und mutmaßlichen Ardspitzen unterscheiden. Während die Knebelfunde aus Asva und Iru mit Pferden als Last- und Nutztiere in Verbindung gebracht werden können (Maldre \& Luik 2009, 41, Abb. 6), gehörten die keilförmigen Geweihgeräte mit trapezförmigem Querschnitt (10-15 cm lang) offenkundig sog. Hakenpflügen an. Form, Größe und Scharfkantigkeit dieser Geräte sowie der an allen auftretende stufenförmige Absatz deutet auf die Funktion als Ardspitze bzw. Schar und zwar in Analogie zu den bekannten ethnographischen und neuzeitlichen Quellen (vgl. Vilkuna 1971). Ilmari Manninen (1933, 51 ff.) zufolge sind noch zu Beginn des 20. Jahrhunderts im Osten der Insel Saaremaa Hakenpflüge vom Typ Ruhnu in Gebrauch gewesen. Diese zeichnen sich durch ihren einfachen Aufbau der Grindel aus, vor allem aber durch das Fehlen der Schar aus Metall (Eisen). Man habe zu dieser Zeit in Gegenden Gotlands, Saaremaa und der nordwestlichen Küstenzone bewusst Aufsätze aus Holz bevorzugt (ebd., Abb. 24, 35). Nicht erwähnt wird, um welche Art von Holz es sich handelte oder ob in der Neuzeit dafür auch Geweih verwendet wurde. Dieser sog. Inseltyp bzw. diese Art von Pflugschar soll indes bestens für steinige Äcker geeignet und auch leichter zu handhaben gewesen sein als gewöhnliche Hakenpflüge. Ein interessanter Aspekt ist weiterhin, dass das Pflügen in bestimmten Teilen der Insel Saaremaa noch während der Neuzeit als Frauenarbeit beschrieben wird (ebd., 52).

Die Funde mutmaßlicher Ardspitzen aus Elchgeweih mit der typischen, einseitig gestuften Keilform beschränken sich indes nicht nur auf Asva, Ridala und Iru allein (Sperling 2006, 103, Taf. XLIX: 4, LVIII: 1-6). Im Material einiger lettischer und litauischer Bronzezeitsiedlungen des Ostbaltikums sind diese Geräte ebenfalls angefunden worden (z. B. Graudonis 1967, Taf. XVIII: 7, 9; Volkaitė-Kulikauskienè 1986, Abb. 21-22). Aus der Verbreitung dieser Geräte

204 Zur jüngsten Funktionsdiskussion der aus tierischen Schulterblättern hergestellten Werkzeuge siehe Luik \& Lang 2010; Sperling 2006, 110. Die Anwendungsmöglichkeiten reichen vom Ährenschneiden bis hin zur Fell- und/oder Lederbearbeitung. 
geht somit hervor, dass die vermuteten Hakenpflüge nicht nur in Bereichen der Alvare auf karstigem Untergrund (Saaremaa, Nordestland) zum Einsatz kamen, sondern in ackerbaulich unterschiedlich geeigneten Böden. In der jüngeren Forschung wird mittlerweile davon ausgegangen, dass sich in Estland bereits zum Beginn des ersten vorchristlichen Jahrtausends eine primitive Form des extensiven Feldbaus mit Zug- und Lasttieren etabliert habe (Lang 2007a, 105 ff.; 2007b, 111 f.). Diskutiert wird indes, ob die Technologie des Pflügens mit Hakenpflug und Zugtier nicht noch früher und zwar im Laufe des zweiten Jahrtausends v. u. Z. in Estland Anwendung fand. In diesem Zusammenhang werden auch die spätneolithisch-bronzezeitlichen Steinbeile und Schaftlochäxte gedeutet, für die neben der Verwendung zum Hacken und Roden der Waldflächen auch eine Funktion als Pflugschar für möglich gehalten wird (siehe Lang 1995a, 159 ff., Abb. 8 f.; Johanson 2006, 27 f.).

Für die Praxis des Ackerpflügens in der Bronzezeit spricht bereits indirekt die Platzwahl der Asva-Siedlungen in den Zonen der fruchtbaren Rendzina-Böden auf Saaremaa (Alvare). Der Pflug könnte sich besonders auf den sandigen Moränenzügen im Osten der Insel, auf denen Asva und Ridala angesiedelt sind, geeignet haben. Die Handhabe des Hakenpflugs hat man sich znächst unter Einsatz von Zugtieren (Asva: Pferd und Rind) und einer von Baumwurzeln befreiten Ackerfläche vorzustellen, wobei der Ard nur die obere Grasnarbe lockert und aufbricht. Im nordestnischen Küstengebiet (Saha-Loo und Proosa) wurden im Rahmen zielgerichtetgroßangelegter Landschaftsarchäologie ganze Areale mit vorgeschichtlichen Feldstrukturen freigelegt und dokumentiert. Gleich mehrere Hektar große Komplexe solcher sog. fossil fields wurden über Feldraine und Feldsteinhaufen erfasst. Bevorzugt wurden auch hier die Zonen der Alvare (Lang 1999, 368). In Umrissen ergeben sich für die planmäßig aneinandergereihten Rechteckparzellen Flächengrößen von 143-920 $\mathrm{m}^{2}$. Diese fossilen Felder vom sog. Baltischen Typ (Baltic oder Pre-Celtic fields) sind in ihrer Entstehung spätestens spätbronzezeitlich (Lang 1995a, 144 ff., Abb. 3-5; 1996, 249 ff.; 2007b, 98 ff.). Die Feldraine und Steinhaufen scheinen gegen den einfachen Hackbau und für ein planmäßiges Pflügen in Kreuzspurtechnik zu sprechen. ${ }^{205}$ Diese archäologische Quellengruppe der fossilen Felder ist im Übrigen auf Saaremaa noch nicht erforscht worden bzw. nachgewiesen.

Zieht man die Beobachtungen der Archäobotaniker zu Rate, so zeigen die Vegetationsanzeiger der Pollendiagramme nicht unerhebliche periodische Übereinstimmungen mit dem archäologischen Quellenbild dieser Region (Nordestland). Die Pollenspektren des Maardu-Sees, in der näheren Umgebung des nordestnischen Saha-Loo, weisen auf menschlich ausgelöste Impulse, die zeitlich durchaus mit den frühen Feldern (Typ Saha-Loo) parallelisieren (Lang 1995a, 163). Für Maardu sind Getreidearten wie Gerste, Weizen und Hafer nachgewiesen. Die dort

205 Pflugspuren sind wiederum nur aus Nordestland bekannt. In Ilumäe II kamen diese unter einer Siedlungsschicht der späten Römischen Eisenzeit zutage. Von deren Zeitstellung im Bereich Neolithikum-Bronzezeit wird ausgegangen (Lang 2003, 156 f., Abb. 44; 2007b, 106 f., Abb. 46). Aus dem lettischen Dievukalns sind Pflugspuren unter der spätbronzezeitlichen Wallanlage aufgetreten und vermutlich bereits mittelbronzezeitlich (Lang 2007a, 67). Zu den möglichen Anwendungen und Vorteilen des Hakenpflugs in Relation zur Bodenart siehe Schier (2009, 36). 
ebenfalls beobachteten Roggenanzeiger gehen eventuell auf eine Art von wilder Begleitvegetation zurück. Diese ganze Auswahl an Süßgräserpollen macht sich dort erst um 700 cal. BC bemerkbar (Lang 1995a, 163; Veski \& Lang 1996, 194 ff., Abb. 1 f.; Saarse et al. 2010, 23 f., Abb. 6). Die Vegetationsanzeiger für Grasund Viehweiden nehmen dann erst wieder mit der ausgehenden Vorrömischen Eisenzeit zu. Der anhand der Holzkohlepartikel in Pollendiagrammen in Erscheinung tretende Brandfeldbau (slash-and-burn cultivation) könnte in Nordestland im Zuge der bronzezeitlichen Spätphase eingesetzt haben, in Analogie zur Entwicklung in Mittelschweden. ${ }^{206}$ Ähnliche Indikatoren für Flächenrodungen und Landkultivierung spiegeln sich auch in den Pollenspektren im südöstlichen Estland. Entsprechende Untersuchungen am Plaani-See zeigen bereits ab 850 cal. BC eine merkliche Zunahme an Cerealia (v. a. Gerste) und Ruderalflora, einhergehend mit einem deutlichen Rückgang der Fichte (Saarse et al. 2010, 21, Abb. 3). Vieles spricht für eine im Laufe der Bronzezeit eintretende, kontinuierlich verlaufende Entwicklung im Agrarsektor.

Was Intensität und Umfang des praktizierten Bodenbaus zur Zeit der AsvaGruppe anbetrifft, so bemerkt V. Lang am Beispiel der untersuchten Feldlappen von Saha-Loo, dass die Anbauflächen der Bronze- und Früheisenzeit wesentlich kleiner gewesen sein müssten als jene in frühgeschichtlichen und mittelalterlichen Perioden mit mindestens drei Hektar Land pro Haushalt. Die archäologischen Anzeichen eines extensiv betriebenen Ackerbaus während der Spätbronzezeit lassen Ernteerträge von eher kleinerer und unregelmäßiger Größenordnung erwarten. Ackerbau wurde also ganz offensichtlich in Ergänzung zur wirtschaftlich dominanten Viehzucht praktiziert (Lang 2007b, 112).

\subsection{Viehzucht}

Das Halten und Züchten von Hausvieh lässt sich auf Saaremaa derzeit bis in das ausgehende Neolithikum zurückverfolgen. Älteste Belege der Rinder- und Schweinezucht stammen vom Fundplatz Loona im Westen der Insel (Maldre 1999, 320 f.). Schaf- und/oder Ziegenhaltung tritt dagegen erstmals mit den Fundplätzen der Asva-Gruppe in Erscheinung. Im Schlacht- und Speiseabfall von Asva und Ridala sind neben Rind (Bos taurus), Schwein (Sus domesticus), Schaf und Ziege (Capra hircus, Ovies aries) erstmalig Pferd (Equus caballus) und Hund (Canis familiaris) nachgewiesen - alles in allem sechs Haustierarten.

In Asva und Ridala konnten insgesamt 4149 bzw. 1554 Knochen Haustieren zugeordnet werden (Maldre \& Luik 2009, 38, Abb. 2). In Iru sind Haustiere ebenfalls in großen Mengen unter den archäologischen Funden (3959 Stück)

${ }^{206}$ Nur ist für das Ostbaltikum nicht jener drastische Rückgang der Kulturanzeiger in der frühen Vorrömischen Eisenzeit festzustellen, wie es für den Westen der Fall ist (Veski \& Lang 1996, 200). Zur Agrartechnik des Brandfeldbaus als Düngemethode in Archäologie und Experiment siehe Schier (2009). Für Estland ist diese in den vorgeschichtlichen Perioden nur in bestimmten Regionen Nordestlands nachgewiesen. 
vertreten, allerdings lassen sich die spätbronzezeitlichen Siedlungsreste schwer von Fundablagerungen jüngerer Perioden trennen (Lang 2007b, 110). Die Siedlungen der Bronzezeit lieferten jedoch mit Abstand mehr Tierknochenabfälle als andere estnische Fundplätze eisenzeitlicher und mittelalterlicher Perioden.

Die Haustierknochen der Siedlungen von Asva, Ridala und Iru machen mengenmäßig ca. 75-80\% des (terrestrischen) Tierknochenmaterials aus (Jaanits et al. 1982, 155; Maldre 1999, 322). Der Wildtierbestand ist dagegen verschwindend klein. Insbesondere die Dominanz der Schaf- und Ziegenknochen ist bemerkenswert, denn es scheint im Ostbaltikum der Bronzezeit Anzeichen für Diversifikationen in Tierzuchtwahl und Ernährungsverhalten zu geben. In den Bronzezeitfundplätzen Lettlands und Litauens verhält es sich nämlich anders: Dort steht der Schaf- und Ziegenanteil deutlich hinter dem der Rinder und Schweine zurück. ${ }^{207}$

Die regionalen Unterschiede im Nachweis der Schweinehaltung eröffnen mögliche Einblicke in die Vegetation der Umgebungen der Siedlungen. Die Schweinezucht müsste durch die Nähe zu Waldweiden begünstigt worden sein, was im Fall Saaremaa indirekt auf die Pflege einiger Baumbestände schließen ließe. Eichenwälder waren in der subborealen Chronozone auf Saaremaa und dem Festland relativ verbreitet. Auch kann davon ausgegangen werden, dass der im Fisch- und Robbenfang anfallende Speiseabfall eine zusätzliche, wenn nicht gar wesentliche Ernährungsbasis der Hausschweine bildete (Lõugas et al. 2007, 28; Maldre 2008, 266).

Leider lässt sich aus den archäologischen Siedlungsbefunden der Asva-Gruppe kaum etwas zu Art und Bedingungen der Tierhaltung aussagen. Lediglich die vergleichsweise schwach ausgebaute Siedlungsbegrenzung könnte ein Hinweis auf die Haltung der Haustiere auf dem umfriedeten Siedlungsareal bedeuten (u. a. im Winter). Das sich in den Tierknochen spiegelnde Verbrauchsbild von Asva und Ridala zeigt bezüglich des Hornviehs ein interessantes Verteilungsbild zugunsten der Schafe/Ziegen gegenüber den Rindern (Tab. 20). Es bleibt schwierig zu beurteilen, ob der Schaf- und Ziegenhaltung der tatsächliche Vorrang in der Viehzucht gegolten haben mochte. Bei Rindern fallen Fleischgehalt und Schlachtgewicht beträchtlich höher aus als bei Schafen bzw. Ziegen, d. h. bei theoretisch ausgewogener Haltung und Nutzung beider Tierarten sind für die Bedarfsdeckung an Fleisch weniger Rindertiere als Schafe oder Ziegen vonnöten, was sich folglich auch im Schlachtund Speiseabfall niederschlagen müsste. Weitere vergleichende Untersuchungen am osteologischen Material anderer Siedlungsplätze, speziell zu den Schlacht- und Ernährungsgewohnheiten der Gemeinschaften, dürften in dieser Frage weiterhelfen. Lembi Lõugas (1994, 75 ff.) und Liina Maldre (2008, 266 ff., Abb. 3-6) konnten am archäozoologischen Material einen vergleichsweise hohen Anteil an Knochen juveniler Tiere nachweisen, doch betrifft dies sowohl Schaf/Ziege als auch Rind.

207 Siehe Brikul̦i, Ķivutkalns, Vinakalns und Narkūnai (Vasks 1994, Tab. 7 f.; Daugnora \& Girininkas 1995, 48, Tab. 1; Maldre 2008, 266). In punkto Größe der untersuchten Ausgrabungsflächen und Mengen des Tierknochenabfalls stehen die estnischen Siedlungen den genannten ostbaltischen deutlich zurück. 
Tabelle 20. Anzahl und Verteilung der Haustierarten auf die Grabungsteile in Asva und Ridala (nach Lõugas 1994; Maldre 2008, 265 f.)

\begin{tabular}{l|c|c|c|c|c|c}
\hline & $\begin{array}{c}\text { Anzahl der } \\
\text { Haustierknochen }\end{array}$ & $\begin{array}{c}\text { Schaf/Ziege, } \\
\%\end{array}$ & $\begin{array}{c}\text { Rind, } \\
\%\end{array}$ & $\begin{array}{c}\text { Schwein, } \\
\%\end{array}$ & $\begin{array}{c}\text { Pferd, } \\
\%\end{array}$ & $\begin{array}{c}\text { Hund, } \\
\%\end{array}$ \\
\hline Asva A-D & 2429 & 51 & 17,9 & 18,8 & 11,8 & 0,5 \\
Asva E & 696 & 37,1 & 36,3 & 14 & 10,6 & 2 \\
Asva F & 973 & 44 & 31 & 15 & 9 & 1 \\
Ridala A & 855 & 39,6 & 21,2 & 29,4 & 15,1 & 2,3 \\
Ridala B & 733 & 56,1 & 14 & 24,1 & 4 & 1,8
\end{tabular}

Ungefähr ein Drittel beider Arten wurde im Jungtierstadium geschlachtet. Das trifft insbesondere auf die Schweine zu: In Ridala sind sogar um die $80 \%$ der ermittelten Schlachttiere jünger als 18 Monate gewesen. Das überwiegend junge Schlachtungsalter bei den Zuchttieren in den Siedlungen der Asva-Gruppe, speziell bei den Rindern, spricht mehr für das Nutzenelement der Fleischgewinnung als für Milch- und Lastvieh. So wurden die meisten Schafe in Ridala nachweislich vor dem zweiten Lebensjahr geschlachtet (Maldre 2008, 268, Abb. 4).

Die Pferde von Asva und Ridala werden als Vertreter einer vergleichsweise kleinen, pony-ähnlichen Rasse angesehen, zumindest sind sie nachweislich kleiner als deren Nachfolger aus wikinger- und jüngerzeitlichen Perioden (Lõugas 1994, 75; Maldre \& Luik 2009, 41). Es gibt Hinweise darauf, dass Bronzezeitmenschen das Pferd nicht nur als potentielles Zugtier (für Hakenpflug), sondern auch als Nahrungslieferanten nutzten. Unter den Tierknochen von Asva (mind. 15 Individuen allein aus Asva A/C) und Ridala (mind. 8) sind viele von Jungtieren. Es gibt allerdings auch vereinzelte höheren Alters (über 4 Jahre) (Lõugas 1992, 26; Maldre 2008, 270; Maldre \& Luik 2009, 40). Ähnliche Beobachtungen wurden im lettischen Brikuli gemacht, wo der große Anteil an Jungtierknochen auf eine Wertschätzung des Pferdefleischs hindeutet (so auch Vasks 1994, 118). Die mutmaßlichen Geweihknebel aus Asva sind gleichzeitig Indiz für die Haltung der Pferde als Zug- und Lasttiere, wenn es auch an diesbezüglichen pathologischen Beobachtungen am Knochenmaterial mangelt (Maldre \& Luik 2009, 41, Abb. 6). Schließlich bleibt die offene Frage, warum ausgerechnet bei den Pferden so unverhältnismäßig viele Zähne im Schlacht- und Speiseabfall von Asva und Ridala auftreten. Bei keiner anderen Tierart ist deren Anteil derart hoch (Maldre 2008, 270).

Beste Einsichten in die Art der Zusammensetzung und Mengenverteilung des osteologischen Fundmaterials von Asva liefert der Grabungsteil F (1965-1966). Den Zählungen von L. Lõugas zufolge (Lõugas 1992; 1994) gehören insgesamt 1828 Tierknochen zum Schlacht- und Speiseabfall (mit Robbenknochen). Die Haustiere machen mit 973 Stück den Großteil (79\%) der Tierknochenfunde aus. Interessant ist das Ergebnis des Versuchs, die Knochenfunde stratigraphisch den beiden Siedlungsphasen von Asva zuzuordnen (Tab. 21). Festzustellen ist zunächst 
Tabelle 21. Haustier- (79\%), Wildtier- (2\%) und Robbenknochen (19\%) aus Asva F, verteilt auf die Häuserbereiche und -horizonte A/C/E und B/D (insgesamt 1237 Stück; Stückzahlen nach L. Lõugas (1994 und mündliche Mitteilung)

\begin{tabular}{|c|c|c|c|c|c|c|c|}
\hline \multirow[t]{2}{*}{ Asva $F(n=1237)$} & \multirow[t]{2}{*}{ Übrige } & \multicolumn{3}{|c|}{ Häuser (Asva II) } & \multicolumn{2}{|c|}{ Häuser (Asva I) } & \multirow{2}{*}{$\frac{\text { Asva I+II }}{\text { A-D }}$} \\
\hline & & $\mathrm{A}$ & $\mathrm{C}$ & $E$ & $\mathrm{~B}$ & $\mathrm{D}$ & \\
\hline Schaf $/$ Ziege $(n=433)$ & 128 & 51 & 95 & 71 & 43 & 45 & 305 \\
\hline Rind $(\mathrm{n}=290)$ & 131 & 28 & 61 & 14 & 27 & 29 & 159 \\
\hline Schwein $(n=149)$ & 52 & 18 & 19 & 18 & 17 & 25 & 97 \\
\hline Pferd $(n=91)$ & 31 & 12 & 25 & 7 & 5 & 11 & 60 \\
\hline \multirow{2}{*}{ Hund $(\mathrm{n}=10)$} & / & / & 3 & 4 & l & 3 & 10 \\
\hline & 342 & 109 & 203 & 114 & 92 & 113 & 631 \\
\hline $\operatorname{Elch}(n=23)$ & 7 & 1 & 4 & 2 & 4 & 5 & 16 \\
\hline \multirow{2}{*}{ Wildschwein $(n=6)$} & / & / & 1 & 3 & / & 2 & 6 \\
\hline & 7 & 1 & 5 & 5 & 4 & 7 & 22 \\
\hline Sattelrobbe $(\mathrm{n}=116)$ & 19 & 27 & 24 & 19 & 2 & 25 & 97 \\
\hline Kegelrobbe $(\mathrm{n}=59)$ & 8 & 22 & 9 & 11 & / & 9 & 51 \\
\hline Ringelrobbe $(n=46)$ & 4 & 19 & 7 & 2 & 3 & 11 & 42 \\
\hline \multirow[t]{2}{*}{ Seehund $(n=14)$} & 1 & 3 & 1 & 2 & / & 7 & 13 \\
\hline & 32 & 71 & 41 & 34 & 5 & 52 & 203 \\
\hline
\end{tabular}

eine Mengenzunahme der Tierknochen, insofern nicht übermäßig sekundär verlagert, in der Häuserphase $\mathrm{A} / \mathrm{C} / \mathrm{E}$ (Asva II). Bemerkenswerterweise geben sich keine nennenswerten Unterschiede in der räumlichen und mengenmäßigen Verteilung der Knochenreste zu erkennen, vielleicht mit Ausnahme im Bereich des Hauses C. Eine innerhalb der Siedlung bevorzugte oder gar gemeinsam genutzte Schlachtstelle lässt sich daraus nicht ableiten. Vieles deutet auf eine nach Hauseinheiten getrennte Verarbeitung und Zubereitung der Fleischbestände hin, zugleich aber auf die große ökonomische Bedeutung der Viehhaltung für die Siedlungssubsistenz.

\subsection{Jagd}

Der Wildtierbestand ist im osteologischen Fundmaterial der Siedlungen der Asva-Gruppe ausgesprochen klein. Für Asva $\mathrm{F}$ und $\mathrm{A}-\mathrm{D}$ sind von größeren potentiellen Jagdtieren nur vereinzelte Knochenfunde nachgewiesen, etwa vom Elch (Alces alces), Wildschwein (Sus scrofa), Braunbär (Ursus arctos), Biber (Castor fiber) und Fuchs (Vulpes vulpes) (Indreko 1939b, 24 ff.; Lõugas 1994, 82). In Asva F beläuft sich die Gesamtzahl aller Wildtierknochen im Schlacht- und Speiseabfall auf nur 46 (2,5\%), in Ridala auf 3\% (Maldre 2008, 272). Die Bedeutung der Wildtierjagd stand ganz offensichtlich klar hinter der Viehzucht und dem Robbenfang zurück, was teils auch mit den naturräumlichen Bedingungen der Insel zu erklären ist (kleine Tierpopulationen, Überjagung etc.). Interessant ist dennoch das geringe Knochenvorkommen vom Elch und anderer Cerviden im 
Schlachtabfall der Siedlungen, zumal sich im Artefaktmaterial ganz andere Anteile abzeichnen. Neben dem Geweih der Wildtiere, diese waren auch durch gezieltes Aufsammeln der Abwurfstangen zugänglich, wurde reichlich Knochenmaterial vom Elch zu Gerät und Schmuck verarbeitet. In Asva und Ridala wurden 16\% bzw. 17\% aller Horn- und Knochenartefakte aus Elchgeweih gefertigt, 4\% bzw. $1-2 \%$ aus Elchknochen. Obwohl die Jagd auf Wild nur sporadisch erfolgte, wurde das Rohmaterial scheinbar ausgiebig genutzt bzw. verarbeitet (Maldre 2008, 271; Luik 2013b). In der Subsistenz der Siedlungen der Asva-Gruppe scheint die Jagd auf Wildtiere eine völlig untergeordnete Rolle gespielt zu haben.

\subsection{Robbenfang}

Bereits die ersten Grabungskampagnen in Asva (1934; 1938) wurden von fachkundigen Untersuchungen am archäozoologischen Material begleitet (Johannes Lepiksaar). Vier Robbenarten - Sattelrobbe, Kegelrobbe, Ringelrobbe und Seehund ließen sich nachweisen (Indreko 1939b, 24). Dem Robbenfang stand somit ein nicht unbedeutender Stellenwert in der Siedlungsökonomie von Asva zu. ${ }^{208}$ Auf Lembi Lõugas gehen die jüngsten Analysen am Tierknochenmaterial der AsvaGruppe zurück, mit genauest möglichen Angaben zur Menge und Verbreitung der Knochen und in Rücksicht auf die jeweiligen Robbenarten (Lõugas 1994, 85 ff., Tab. 12-14; Storå \& Lõugas 2005, 99 f., Tab. 1).

Asva und Ridala stellen die bislang einzigen archäologisch untersuchten Fundstellen im östlichen Ostseeraum dar (Neolithikum-Bronzezeit), in denen sich subfossile Überreste aller vier Robbenspezies zusammen fanden (Tab. 19; vgl. Storå 2001, 25 f., Tab. 7). Dabei ließen sich im Siedlungsabfall von Asva (alle Grabungsteile) insgesamt 721 Knochen- und Zahnfragmente der genannten Robbenarten ermitteln. Anteilig macht dies ca. 39\% am gesamten Tierknochenmaterial aus. In Ridala (A und B) wurden 377 (19\%) Robbenknochen gezählt (Storå \& Lõugas 2005, Tab. 1, 3; Maldre 2008, 272). Aus Kaali sind 39 Stück überliefert (nur Sattel- und Kegelrobben).

Das Knochenmaterial erweist sich indes als sehr fragmentarisch, so dass kaum verlässliche Größen- und Altersangaben zu den in Asva zerlegten Robbentieren möglich sind. Beobachtungen zu Jagdgewohnheiten und -zielen (Jagdsaison, bevorzugtes Jagdalter der Robben) sind somit nur eingeschränkt zu erhalten. Zugleich ist der Mengenanteil der artenspezifisch nicht bestimmbaren Knochen

208 Auf eine gewisse Bedeutung des Fischfangs für die Siedlungsökonomien von Asva und Ridala wurde wiederholt hingewiesen (Indreko 1939b; Lõugas 1999; Maldre 2003), doch liegen noch wenig ausgewertete Daten und Informationen zum Fischknochenspektrum im Speiseabfall der Siedlungen vor. In den gegenwärtig laufenden Grabungskampagnen von Asva G werden von Lembi Lõugas die Fischknochenfunde mittels Flotation aus dem Erdmaterial gesiebt und untersucht - auch in der Frage, warum bislang nur Süß- oder Brackwasserfische (Hecht, Barsch, Plötze) und keine Meeresfische (Dorsch, Flunder und Baltischer Hering) nachgewiesen werden konnten (dazu Sperling et al. 2013). 
Tabelle 22. Die Robbenarten (Knochenanzahl) in den Siedlungen (nach Storå \& Lõugas 2005, Tab. 1, 3)

\begin{tabular}{l|l|r|r|r}
\hline \multicolumn{1}{c|}{ Spezies } & \multicolumn{1}{c|}{ Lat.; engl. } & $\begin{array}{c}\text { Asva } \\
\text { A-F } \\
(665)^{\mathbf{2 0 9}}\end{array}$ & $\begin{array}{c}\text { Ridala } \\
\text { A-B } \\
(379)\end{array}$ & $\begin{array}{c}\text { Kaali } \\
(39)\end{array}$ \\
\hline Sattelrobbe & $\begin{array}{c}\text { Pagophilus groenlandicus; } \\
\text { harp seal }\end{array}$ & 44 & 28 & 2 \\
Kegelrobbe & Halichoerus grypus; grey seal & 32 & 22 & 14 \\
Seehund & Phoca vitulina; harbour seal & 12 & 6 & $/$ \\
Ringelrobbe & Phoca hispida; ringed seal & 8 & 2 & $/$ \\
Unbestimmte & & 569 & 321 & 23
\end{tabular}

sehr hoch (Tab. 22). Welche der Robbenarten bevorzugt gejagt oder ausgebeutet wurden, lässt sich daher nicht feststellen. Zumindest sind Sattel- und Kegelrobbe am häufigsten im Schlachtabfall vertreten. Der vergleichende Blick auf die sog. Robbenfängerstation von Otterböte zeigt, je nach Erhaltung der Knochenreste und der Bestimmungsmethoden, wie viel an Beobachtungen zu Jagd- und Fanggewohnheiten sowie der Art der Nutzung der Tiere getroffen werden können. Im bronzezeitlichen Schlachtabfall auf Åland gehört ein Großteil der Robbenknochen juvenilen Kegelrobben an (unter einem Jahr). Immerhin ist dies hinweisgebend bezüglich der unternommenen Jagden während der kurzzeitigen Brut- und Aufzuchtperioden der Robben. Gleichzeitig weist dies auf eine mutmaßliche Präferenz der Jungtiere in Bezug auf deren Fettreserven (Tran), Fleischanteile und Lederhäute (Storå \& Lõugas $2005,99,104)$.

Im Ostseeraum stellte die Jagd auf Robben bereits seit dem mittleren Neolithikum eine wichtige Wirtschaftsquelle der prähistorischen Gemeinschaften dar (Storå 2001). Auf Saaremaa sind Sattelrobben und Kegelrobben bereits in zwei neolithischen Siedlungen (Loona, Naakamäe) nachgewiesen (Lõugas 1999, 193, Tab. 3). Diese beiden Robbenarten dominieren sowohl das osteologische Fundmaterial von Asva als auch das der Bronzezeit-Siedlungsplätze auf den Åland-Inseln (Storå \& Lõugas 2005, Tab. 2). Heutzutage haben sich Sattelrobben und Kegelrobben in nördliche Bereiche der Ostsee zurückgezogen, sind ansonsten vornehmlich in subarktischen und kalttemperierten Zonen des Atlantiks anzufinden. ${ }^{210}$

Von den Sattel- und Kegelrobben ist bekannt, dass diese alljährlich zum Winterende (ab Ende Februar) ihre Brutplätze auf Packeisflächen anzulegen pflegen und zwar stets in sicherer Nähe zum offenen Wasser. Bei milden Wintern

$209 \mathrm{Zu}$ den 665 ermittelten Robbenknochen werden noch 56 Stück der kurzlebigen und fundarmen Phase Asva III (mittlere bis jüngere Eisenzeit) zugeordnet (Storå \& Lõugas 2005, Tab. 1). Meist handelt es sich dabei um Oberflächenfunde, die auch gut sekundär verlagerte Siedlungsfunde sein können. Es ist nicht erwiesen, ob in Asva später überhaupt Robbenfang betrieben wurde.

${ }^{210}$ Kegelrobben und Ringelrobben indes gehören zu den Seehundarten, die noch bis in die Neuzeit regelmäßig in Küstenbereichen des Bottnischen und Finnischen Meerbusens gejagt wurden (Lehtonen 1971, 182, Karte 1, 2). Einige Populationen von Ringelrobben haben noch heute Brutplätze in verschiedenen Küstengegenden des Finnischen Meerbusens und der Rigaischen Bucht. 
ohne geeignete Eisdecken werden dann auch Brutplätze auf Schären oder in höher gelegenen, wellengeschützten Strandgegenden aufgesucht. Für die Schärengebiete in Mittelschweden (Stockholmer Gegend), auf den Åland-Inseln und auf dem westlichen Teil von Saaremaa ist dies hinlänglich dokumentiert (Gustavsson 1997, 111; BACC 2008, 344). Die Jungtiere der Sattelrobben verlassen das Eis nicht in ihren ersten sechs Lebenswochen, für Jäger mochten sich in dieser Zeit beste Fangmöglichkeiten geboten haben. Die Herden der Sattel- und Kegelrobben pflegen, dem allmählichen Rückzug der Eisflächen folgend, weite Gebiete abzuwandern. Die Ringelrobben dagegen sind ganzzeitlich ortsgebunden.

Von Ringelrobben und Seehunden wird angenommen, dass diese seit dem späten Holozän in Regionen der Åland-Inseln und Westestlands eher seltene und unregelmäßige Besucher darstellten. Unsicher ist noch immer, ob diese Robbenarten in der östlichen Ostsee Brutstätten hielten (Storå \& Lõugas 2005, 102). Während die Seehunde ihre Jungen während der Sommermonate aufzogen, waren die Ringelrobbenjungen vorwiegend im Spätwinter zu jagen. Die Ringelrobben legen, anders als die Sattel- und Kegelrobben, ihre Aufzuchtplätze in oberirdischen Schneenestern und in direkter Nähe zu selbst geformten Eis- und Luftlöchern an. Die Verstecke der Robbentiere in den sog. Koben im Schnee sind stets gut getarnt und neuzeitliche Quellen wissen von Robbenfängern zu berichten, die deshalb ihre Hunde auf Jagden mitzunehmen pflegten um diese aufzuspüren (Lehtonen 1971, 184). Im Tierknochenspektrum von Asva und Ridala sind Hunde bekanntlich nachgewiesen, so dass sich ein möglicher Zusammenhang dieser Haustiere mit dem Robbenfang auftut. Anderen Quellen zufolge sollen bei solchen Fangaktionen nur vergleichsweise wenige Tiere erschlagen oder harpuniert worden sein. Massenfänge auf dem Eis waren bei Ringelrobben nicht möglich (Gustavsson 1997, 111 f.; Storå 2001, 46).

Kenneth Gustavsson (1997, 112, 115 ff.) beschreibt die Abhängigkeit des Jagderfolgs bei winterlichem Robbenfang im nördlichen Ostseeraum von der jeweiligen Jahrestemperatur und der Beschaffenheit der Eisdecke. Bei besonders kalten Winterbedingungen können sich die Robbengruppen auf küstenferne und für den Menschen gefahrvolle Wegdistanzen zurückziehen. Milde Winter mit schnellem Eisrückgang oder gar driftenden Eisschollen indes könnten die Robben zu nächster Nähe zum Land bzw. zur Küste zwingen. Mit vergleichsweise wenig Arbeits- und Zeitaufwand ließe sich unter solchen, wenn auch freilich selten eintretenden Bedingungen reichlich Beute machen. Unabhängig von Gustavssons Überlegungen geht Jan Storå $(2001,46)$ davon aus, dass im Ostseegebiet während der Jungsteinzeit Jagden vorwiegend in eisfreien Perioden, d. h. in Frühlingsund Sommermonaten, unternommen wurden. Auch vermutet er Brutplätze der Sattelrobben in schwer erreichbaren Lagen weitab der Küsten (u. a. auf Treibeis). Ausgehend von seinen osteologischen Analysen am Schlachtabfall verschiedener neolithischer Siedlungen sei der Jagdmodus in vorgeschichtlichen Zeiten (v. a. Neolithikum) auf eine begrenzte, aber kontrollierte Beutezahl und über längere Perioden hinweg ausgerichtet gewesen. Somit stellt sich auch die Frage nach dem Verhältnis zwischen Aufwand und Nutzen der Robbenjagd im Neolithikum und 
in der Bronzezeit und ob die ständige Beutemaximierung im Robbenfang wirklich ein erstrebtes Ziel der Jäger darstellte. Man war sicherlich darauf bedacht, die Tierpopulationen nicht drastisch zu minimieren und zugleich das Gefahrenrisiko der Jagd niedrig zu halten. Dennoch fragt sich, wie groß die für die ökonomische Gemeinschaftssubsistenz erforderlichen Beutemengen gewesen sein könnten.

Versuche, einige der archäologischen Funde der Asva-Gruppe mit dem Robbenfang in Verbindung zu bringen, erwiesen sich bislang als hypothetisch. Das betrifft zunächst die keramischen ,Trangefäße' (siehe Kap. 8.8.1). Bei den sog. Harpunen ist diese Verwendung ebenfalls fraglich (Abb. 121: 1-2). Um die zähe Lederhaut der Robbentiere zu durchdringen, wird eher eine schmale, angespitzte Jagdwaffe erwartet. Indreko indes bezeichnete diese Geräte als Seehundstechgabeln, ohne jedoch deren Funktion zu erläutern (1939b, 24, Abb. 7: 1; 1961, 420, Abb. 1: 5). Er bezog sich ganz offensichtlich auf das aus der Ethnographie bekannte ,Stechen' der Robben - eine Methode, die noch in Verbindung mit dem Robbenfang moderner Zeiten Erwähnung findet (Lehtonen 1971, 183). Durch gezieltes Verstopfen der Eisluftlöcher zwang man die Robben zum Luftschnappen an die Oberfläche einer der wenigen ausgesuchten Öffnungen und stach ihnen bei sich bietender Gelegenheit die Harpunenspitze in den offenen Rachen. Das abtauchende und bald ermüdende Tier mit der losen, an einem Strick befestigten Harpune im Körper konnte dann unter dem Eise hervorgezogen werden. Ansonsten kamen bei den Jagden, den ethnographischen und archäologischen Belegen zufolge, vornehmlich Fangnetze zum Einsatz (Manninen 1931, 79 f.; Storå 2001, 46 f.).
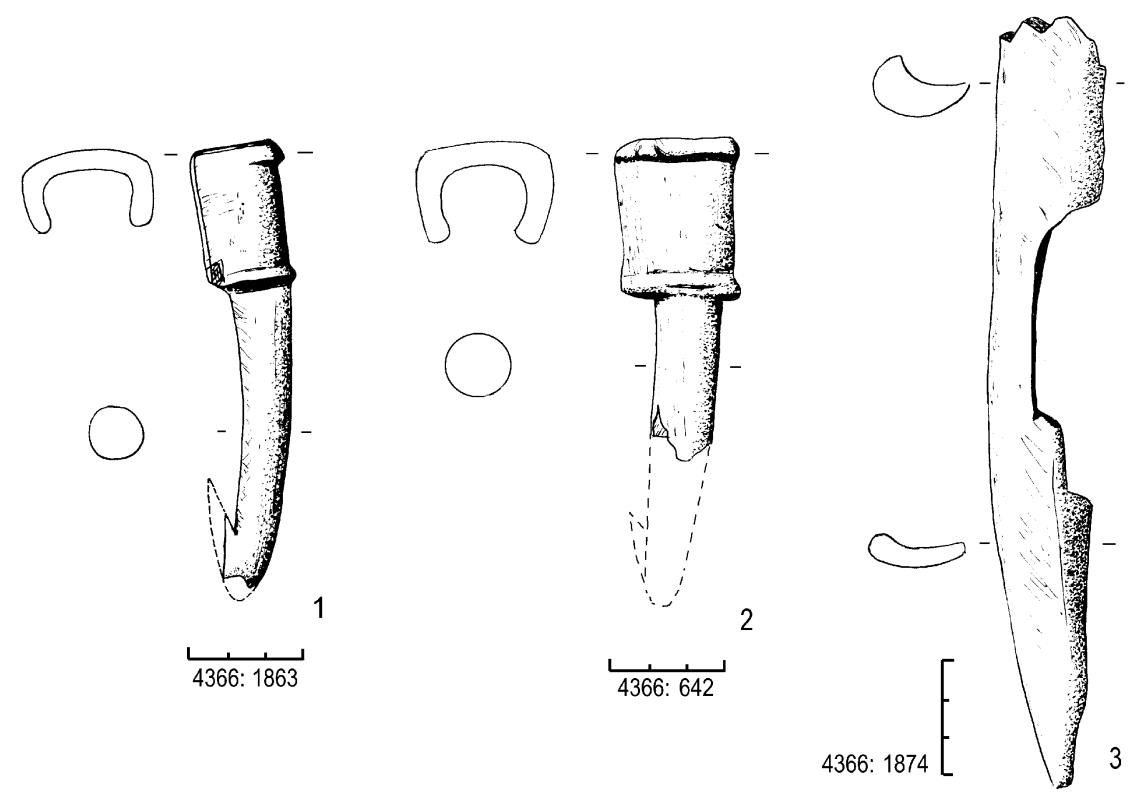

Abb. 121. Sog. Seehundstechgabeln aus Asva F (nach Sperling 2006, Taf. XLVIII: 3-5). 
Zur Bedeutung des Seehundfangs für die betreffenden Siedlungsökonomien der Bronzezeit sind Gustavssons Ausführungen zur Robbenjagd auf den ÅlandInseln (Kökar-Schäre) zu Rate zu ziehen (Gustavsson 1997, 112 ff.; mit weiterer Literatur): Die aus Robbentieren zu gewinnende Produktpalette des Menschen ist ausgesprochen breit, sie reicht vom Fleisch der Robben über deren Fell bis zum Tran. Die Möglichkeiten der Verarbeitung der tierischen Bestandteile sind nicht minder vielseitig. Laut ethnographischen Quellen (u. a. für Kökar und Umgebung) wurde sowohl das Fleisch (inklusive Innerereien, Blut) als auch der Tran (u. a. als Butterersatz) zubereitet und verzehrt. Dabei schien stets das Fleisch der Jungtiere bevorzugt worden zu sein. Ausgewählten Hautpartien der Robben (mit Tran) wurde sogar eine gewisse medizinische Heilkraft zugesprochen, weshalb sie im fennoskandischen Raum der Neuzeit ein weit gefragtes, überregional verhandeltes Produkt darstellten. Auch wusste man stets das Fell der Robben mit zu nutzen und, je nach Verwendungsart und Verarbeitungsmethode, Kleidung, Schuhwerk (bzw. Sohlen) und Handschuhe zu fertigen. Längste Zeit waren vor allem die Fellbzw. Lederprodukte ihrer flexiblen, reißfesten und wasserfesten Eigenschaften wegen begehrt. Abgesehen von Mützen, Taschen oder Sammelbehältern können aus dem Robbenleder äußerst elastische, belastbare Seile und Taue hergestellt werden, die lange Zeit im Schiffsbau oder im Fischfang (u. a. für Netze) Anwendung fanden. Interessanterweise sollen sich die Felle von Jungtieren besonders gut verarbeiten lassen und ausgesprochen wasserfest sein. Schließlich wird das Robbenfett, der Tran (engl. blubber), zu den wertvollsten, da ebenso vielseitig einsetzbaren Produkten gezählt. Zunächst ließ sich daraus eine Art von Teer gewinnen, der als wasser- und windabdichtendes Baumaterial verwendet werden konnte. Auch kennt man die Verarbeitung und Verwendung des Robbenfetts als Lampenöl. Der Tran dürfte bereits in vorgeschichtlichen Perioden zu einer unverzichtbaren Licht- und Wärmequelle für die Menschen avanciert sein. Für das bronzezeitliche Ostbaltikum fehlt es noch an archäologischen Indizien für eine Verwendung des Trans als Lampenöl (z. B. Gefäßrückstände). Trotzdem ist davon auszugehen, dass die Gemeinschaften der Asva-Gruppe regen Gebrauch vom Tranöl machten (u. a. als Lampen). ${ }^{211}$

Angesichts dieser vielseitigen Verwendungsmöglichkeiten ist die Bedeutung des Robbenfangs für die Siedlungsökonomien der Asva-Gruppe nicht hoch genug einzuschätzen. Die dabei anfallenden Produkte waren nicht nur lebenserhaltend, sie steigerten auch erheblich das Lebensniveau der Gemeinschaften. Es ist gut denkbar, dass sich bei den Menschen der Asva-Gruppe gute Absatzmöglichkeiten für Tran-, Fleisch- und Lederprodukte, auch überregional, boten. Dahin weisen die wetter- und naturbedingten Risiken der Beschaffung, zumal die Jagden auf Robben gefährliche Unternehmungen für Leben und Existenz der Menschen und Gemeinschaften darstellten. Die beschriebenen Aktivitäten auf dem Agrarsektor,

211 Solche sind bereits aus der Ertebølle-Kultur bekannt und werden in den keramischen Ovalschalen aus der neolithischen Siedlung im lettischen Sārnate vermutet (Bērziṇš 2008, 162 ff., Abb. 39; spätes Sārnate, ca. 3630-2850 cal. BC). 
eingedenk des vielseitig verarbeitenden Handwerks, mögen theoretisch ausreichend gewesen sein für eine autark funktionierende Siedlungsökonomie. Das wird zumindest aus dem Vergleich mit anderen Siedlungen der Asva-Periode in benachbarten Regionen deutlich, bei denen eine vergleichbar zusammengesetzte Subsistenz wie die von Asva lediglich von der Wildtierjagd ergänzt wurde (siehe Vasks 1994). Der hohe ökonomische Stellenwert des Robbenfangs erschließt sich uns nicht nur durch den hohen Tierknochenanteil im Schlachtabfall der Siedlungen allein. Dahinter steht noch der Zeit- und Arbeitsaufwand der Beschaffung. Gustavsson hat die lebensbedrohlichen Risiken der Fangfahrten beschrieben (auch Indreko 1939b, 24). Ungünstige Wetter- und Eisbedingungen konnten weite, küstenferne Fahrten über mehrere Wochen erfordert haben (Manninen 1931, 88 f.; Gustavsson 1997, 111 ff.). Zudem hat man sich die Robbenfangaktionen in Gruppen organisiert, mit mehreren beteiligten Personen, vorzustellen - auch ein nicht unwesentlicher Risikofaktor im alltäglichen Kampf um die Existenzsicherung der bronzezeitlichen Lebensgemeinschaften. Nichtsdestotrotz scheinen die Aussichten auf Erfolg und Ausbeute derlei Unternehmungen motiviert und gewissermaßen entschädigt zu haben, so dass die wirtschaftliche Bedeutung der Robbenjagd für die Siedlungssubsistenz allein aus diesem Aspekt heraus den anderen Wirtschaftsweisen vorangestellt werden müsste. Gerade vor dem Hintergrund der mit den Fangfahrten verbundenen Kontakt- und Austauschmöglichkeiten und der angesprochenen Fremdbezüge in der örtlichen Keramik und den Gießerfunden mag sich der Robbenfang und die Verarbeitung der dabei anfallenden Tierprodukte zu einem Wirtschaftszweig mit verlockenden überregionalen Absatzmöglichkeiten entwickelt haben. Interessant scheint deshalb der Gedanke, die regional exklusive Erscheinung der Knickwand- und Henkelschalen und der intensiven Bronzeverarbeitung in der Asva-Gruppe in direkter Verbindung mit einer spezialisierten Robbenfangtätigkeit zu sehen. 